\title{
Narrow Magnonic Waveguides Based on Domain Walls
}

\author{
Felipe Garcia-Sanchez, ${ }^{1, *}$ Pablo Borys, ${ }^{2}$ Rémy Soucaille, ${ }^{1}$ Jean-Paul Adam, ${ }^{1}$ Robert L. Stamps, ${ }^{2}$ and Joo-Von Kim ${ }^{1, \dagger}$ \\ ${ }^{1}$ Institut d'Electronique Fondamentale, UMR CNRS 8622, Université Paris-Sud, 91405 Orsay, France \\ ${ }^{2}$ SUPA, School of Physics and Astronomy, University of Glasgow, Glasgow G12 8QQ, United Kingdom
}

(Received 17 November 2014; revised manuscript received 1 May 2015; published 18 June 2015)

\begin{abstract}
The channeling of spin waves with domain walls in ultrathin ferromagnetic films is demonstrated theoretically and through micromagnetics simulations. It is shown that propagating excitations localized to the wall, which appear in the frequency gap of bulk spin wave modes, can be guided in curved geometries and propagate in close proximity to other channels. For Néel-type walls arising from a DzyaloshinskiiMoriya interaction, the channeling is strongly nonreciprocal and group velocities can exceed $1 \mathrm{~km} / \mathrm{s}$ in the long wavelength limit for certain propagation directions. The channeled modes represent an unusual analogy of whispering gallery waves that are one dimensional and nonreciprocal with this interaction. Moreover, a sufficiently strong Dzyaloshinskii-Moriya interaction can create a degeneracy of channeled and propagating modes at a critical wave vector.
\end{abstract}

DOI: 10.1103/PhysRevLett.114.247206

Surfaces and boundaries are natural elements along which propagating waves can become localized. A wellknown example concerns whispering gallery modes, which represent a class of eigenmodes in which multiple reflections along a concave boundary lead to localized waves that propagate freely along curved surfaces. Such channeled modes appear in a wide variety of contexts, ranging from the original observation by Lord Rayleigh of whispering gallery sound waves in the rotunda of St. Paul's Cathedral [1] to guided light in nanometer scale dielectric resonators based on zinc oxide needles [2]. In ferromagnetic systems, spin waves can be localized along film surfaces due to the dipolar interaction. Such excitations are known as DamonEshbach modes and represent long wavelength spin waves in the magnetostatic limit. We show here that spin wave excitations localized to a domain wall can have unexpected and previously unrecognized features. One consequence is that we can view these as an unusual analogy to whispering gallery modes with features not found in other systems due to a breaking of chiral symmetry.

In the context of magnonics [3-7], where the control of spin waves is sought as a practical means of transmitting and processing information (in the same vein as the control of light in photonics), the capacity to propagate spin waves along curved paths is essential for any form of circuit design and is crucial for wave processing schemes that rely on spin wave interference $[8,9]$. While Damon-Eshbach modes are strongly localized for films above hundreds of $\mathrm{nm}$ in thickness, they are less useful for guiding spin waves in nanostructured materials and ultrathin films. Propagation along curved wires has been demonstrated experimentally with the assistance of current-induced Oersted fields [10] that minimize scattering [11], but it remains unclear whether such schemes are feasible in complex magnonic circuits. Moreover, wave packet dispersion can be problematic for
PACS numbers: 75.30.Ds, 75.40.Gb, 75.78.Fg, 85.70.Kh

maintaining coherence over distances of several microns, since ferromagnetic spin waves are largely dispersive, particularly at shorter wavelengths at which the exchange interaction dominates. Finally, issues related to lithography and nanofabrication, such as edge roughness or variability in device dimensions, may become prohibitive for reproducible spin wave properties at sizes below $100 \mathrm{~nm}$.

Here, we present a paradigm for spin wave propagation that relies on magnetic domain walls as natural waveguides. It is well established that spin waves propagating across a domain wall experience a scattering potential, which for static Bloch-type walls is reflectionless [12,13] and only leads to phase shifts $[8,14]$ but can result in momentum transfer for dynamic walls [15-17]. Phenomena related to the latter have motivated studies examining how domain wall motion can be effected by spin waves alone [18-22]. Instead, we focus on a class of eigenmodes localized to the domain wall center but which propagate freely in the direction parallel to the wall as a result of a confining potential. We show theoretically and through micromagnetics simulations that such modes can be channeled in curved geometries with no additional scattering, particularly for excitation frequencies in the gap of bulk spin wave modes. These modes are distinct from other known examples of whispering gallery modes because they are one dimensional. Moreover, chiral symmetry breaking due to a Dzyaloshinskii-Moriya interaction (DMI) leads to nonreciprocal propagation along Néel walls, a feature unknown for whispering gallery modes.

The basic principle of the domain wall magnonic waveguide is illustrated in Fig. 1. Consider a thin rectangular ferromagnetic wire with dimensions of $1000 \times 250 \times 1 \mathrm{~nm}$ and a perpendicular magnetic anisotropy along the $z$ axis [23]. A domain wall separates two uniformly magnetized "up" and "down" states with the wall axis along $y$, which is perpendicular to the wire axis $x$. The spin waves considered 


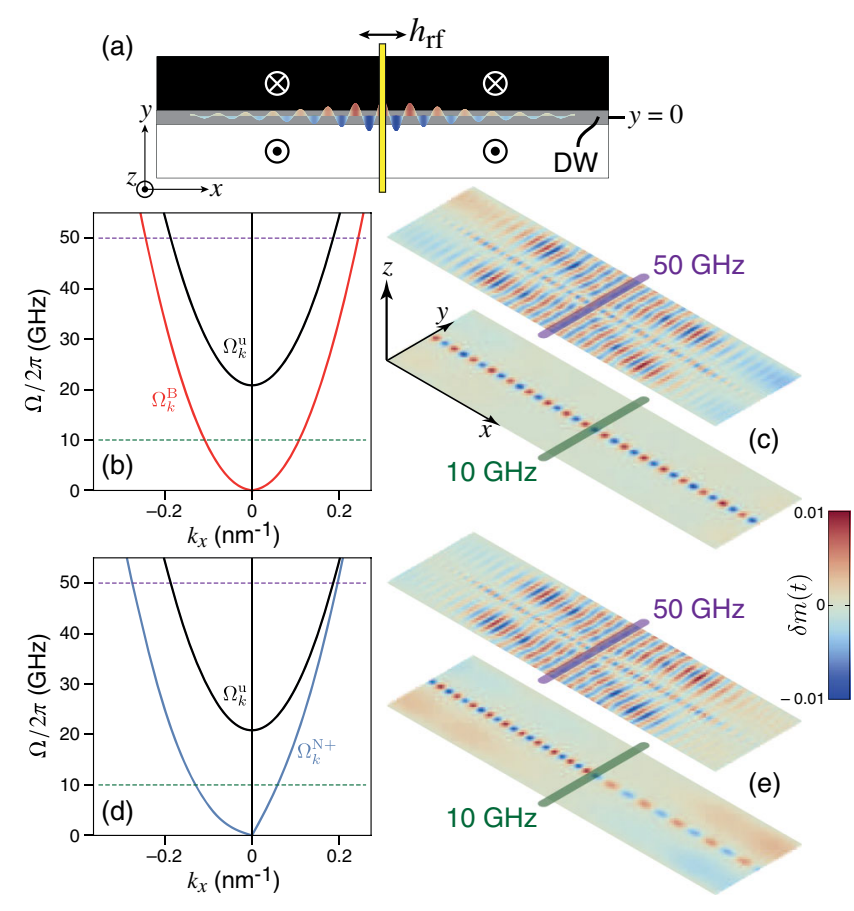

FIG. 1 (color online). A magnonic waveguide based on a domain wall (DW). (a) Geometry for spin wave propagation along the center of the wall, where a radio frequency antenna generating an alternating field $h_{\mathrm{rf}}$ excites spin waves that propagate along the $(x)$. (b), (d) Dispersion relation for channeled Bloch [(b), red curve], $\Omega_{k}^{B}$, and Néel [(d), blue curve], $\Omega_{k}^{N}$, domain wall spin wave modes in comparison with bulk spin waves (black curve), $\Omega_{k}^{u}$. For the Néel wall case, $D=1.5 \mathrm{~mJ} / \mathrm{m}^{2}$. (c),(e) Simulation results of propagating modes for excitation field frequencies in the bulk $(50 \mathrm{GHz})$ and in the gap $(10 \mathrm{GHz})$ for Bloch (c) and Néel (e) walls. These driving frequencies are shown as dashed lines in (b),(d).

are associated with localized domain wall eigenmodes that propagate along the $x$ direction, parallel to the domain wall. In the micromagnetics simulations used [23,26], these modes are driven by a microwave antenna that is modeled as a line source of a sinusoidal excitation field $h_{\mathrm{rf}}$.

In the presence of an isotropic exchange and dipoledipole interactions, the Bloch-type domain wall minimizes the volume dipolar interaction and it is characterized by moments that rotate in a plane $(x z)$ perpendicular to the wall direction $(y)$. For this wall type, there exists a family of spin wave eigenmodes,

$$
\psi_{k}(x, y, t)=\exp \left[i\left(\Omega_{k}^{B} t-k_{x} x\right)\right] \operatorname{sech}(y / \lambda),
$$

which are localized in the direction perpendicular to the domain wall $(y)$ on a length scale $\lambda$ but propagate as plane waves parallel to the domain wall $(x)$ [12]. Here, $\lambda=$ $\sqrt{A / K_{0}}$ represents the characteristic wall width parameter where $A$ is the exchange, $K_{0}=K_{u}-\mu_{0} M_{s}^{2} / 2$ is the effective perpendicular anisotropy constant, and $M_{s}$ is the saturation magnetization. These modes are exchangedominated spin waves whose dispersion relation is

$$
\Omega_{k}^{B}=\sqrt{\omega_{k}\left(\omega_{k}+\omega_{\perp}\right)},
$$

where $\omega_{k}=2 \gamma A k_{x}^{2} / M_{s}$ is the quadratic exchange part, $\omega_{\perp}=2 \gamma K_{\perp} / M_{s}$, and $K_{\perp}=\mu_{0} N_{y} M_{s}^{2} / 2$ is a transverse anisotropy that represents the dipolar interaction due to volume charges at the domain wall center, with $N_{y}$ representing an effective demagnetization constant along the wall axis $y$. For ultrathin films $N_{y} \approx d /(d+\pi \lambda)$, where $d$ is the film thickness [27]. These modes are gapless because the effective field associated with the perpendicular anisotropy cancels out at the wall center. In Fig. 1(b), the dispersion relation Eq. (2) is shown in comparison with the usual bulk spin wave modes for the uniformly magnetized state,

$$
\Omega_{k}^{u}=\omega_{k}+\frac{2 \gamma K_{0}}{M_{s}},
$$

where we have assumed $A=15 \mathrm{pJ} / \mathrm{m}, K_{u}=1 \mathrm{MJ} / \mathrm{m}^{3}$, $M_{s}=1 \mathrm{MA} / \mathrm{m}$, and $d=1 \mathrm{~nm}$. For a microwave field excitation in the frequency gap of the bulk modes $\Omega_{k}^{u}$, which is determined by $2 \gamma K_{0} / M_{s}$, one observes that only the localized Winter modes $\Omega_{k}^{B}$ are excited and are effectively channeled along the domain wall center [Fig. 1(c), excitation at $10 \mathrm{GHz}$, which acts as a local potential well for the spin waves. The wavelength at $10 \mathrm{GHz}$ is approximately $60 \mathrm{~nm}$, which means there is subwavelength confinement in both the film thickness $(1 \mathrm{~nm})$ and across the width of the domain wall $(\pi \lambda \approx 18 \mathrm{~nm})$; such localized modes therefore represent true one-dimensional propagation of spin waves. When the microwave field is applied in the frequency band of the bulk modes, the channeling phenomenon is preserved whereby the localized modes can be seen to propagate with a higher wave vector than the bulk modes [Fig. 1(c), excitation at $50 \mathrm{GHz}$ ].

For ultrathin ferromagnetic films on substrates with a large spin-orbit coupling, an interfacial DMI [28-31] can appear that favors a Néel-type domain wall $[32,33]$. An example of such a material system is the asymmetric $\mathrm{Pt} / \mathrm{Co} / \mathrm{Al}_{2} \mathrm{O}_{3}$ multilayer, where the ultrathin Co layer harbors left-handed Néel walls at equilibrium [34] and possesses a strong interfacial DMI [35] that is consistent with the $D$ values used here. The moments in this wall type rotate in a plane $(y z)$ parallel to the wall direction $(y)$, which leads to an increase in the volume dipolar interaction but which is subsequently compensated by the DMI above a critical value, $D>D_{c}$ [33]. In this case, the inclusion of the DMI leads to a hybridization of the Winter modes [36]. Nevertheless, an expression for the channeled mode frequencies for Néel-type walls can be found from perturbation theory by using the Winter modes in Eq. (1) as a scattering basis, which involves computing frequency shifts due to terms such as $\left\langle\psi_{k}|\operatorname{sech}(y / \lambda)| \psi_{k}\right\rangle$ and $\left\langle\psi_{k}\left|\operatorname{sech}(y / \lambda) \partial_{x}\right| \psi_{k}\right\rangle$ [36]. The resulting eigenfrequencies are 


$$
\Omega_{k}^{N \pm}=\sqrt{\omega_{k}\left(\omega_{k}-\omega_{\perp}+\frac{\omega_{D, k}}{k_{x} \lambda}\right)} \pm \omega_{D, k},
$$

where $\omega_{D, k}=\pi \gamma D k_{x} / 2 M_{s}$. In addition to an ellipticity in the precession, the DMI results in a linear wave vector dependence for the mode frequency, which is consistent with behavior in other geometries [36-39]. However, this linear $k_{x}$ dependence does not lead to a simple shift in the quadratic dispersion relation as a result of the ellipticity. Instead, dispersion relation becomes markedly asymmetric with respect to $k_{x}=0$ [Fig. 1(d)], where a quasilinear variation is seen for $k_{x}>0$ while a strongly quadratic variation is preserved for $k_{x}<0$. This asymmetry leads to pronounced differences in the left- and right-propagating wave vectors, which can be seen for microwave field excitations in the frequency gap and in the frequency band of the bulk spin wave modes [Fig. 1(e)]. The channeling properties of the Néel-type wall are preserved even in cases where the localized and propagating mode frequencies are closely spaced, which can be seen for the $k_{x}>0$ propagation at around $50 \mathrm{GHz}$ in Fig. 1(e). Note that in the limit of $k \rightarrow 0$ and $\omega_{\perp} \rightarrow 0$, Eq. (4) predicts an instability in the domain wall ground state $\Omega_{k}^{N-}=$ 0 for a critical value of the DMI, $D_{c 2}=4 \sqrt{A K_{0}} / \pi$, which is consistent with previous work $[32,33]$. Our simulations indeed show that straight domain walls become unstable for $D>D_{c 2}$. It is interesting to note that the energies of the channeled and bulk mode become degenerate for a certain value of $k_{x}$ for finite $D$. This value of $k_{x}$ represents an inversion of the gap separating the localized from the bulk states.

To verify the perturbation theory and to explore simultaneous propagation along multiple channels, the spin waves were studied using micromagnetics simulations for a threedomain structure comprising two parallel domain walls [23]. The simulated dispersion relations for $k_{x}>0$ propagation in this configuration are presented in Fig. 2. Both Bloch $(D=0)$ and Néel-type walls $\left(D=3 \mathrm{~mJ} / \mathrm{m}^{2}\right)$ were considered. For all channeled domain wall and bulk modes, the analytical theory gives a good account of the simulated dispersion relations, where small discrepancies arise due to limited wave vector resolution resulting from the finite size of the simulation grid. For the Néel wall case, we observe two frequency branches since the propagation in the two domain wall channels occurs for different relative chiralities of the domain wall. No discernible interference is observed between the two channels, which indicates that the wall modes can propagate with distinct wave vectors along the different channels with minimal "crosstalk." An interesting property of these Néel wall branches is that the difference in their frequencies, $\Delta \Omega_{k}^{N}=\Omega_{k}^{N+}-\Omega_{k}^{N-}=2 \omega_{D, k}$, is simply proportional to $D$. Therefore, a simultaneous measurement of these two branches allows the DMI strength to be probed.

Spin waves guided using domain walls in curved geometries are shown in Fig. 3. A $200 \mathrm{~nm}$ wide curved track with a $90^{\circ}$ bend is considered, with a radius of curvature of approximately $1600 \mathrm{~nm}$ for the outer edge [23]. The

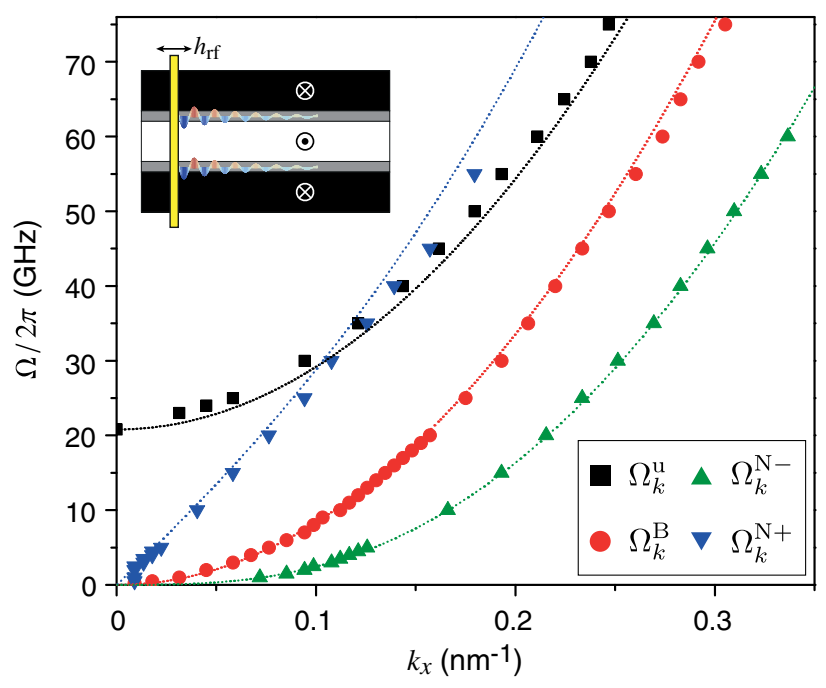

FIG. 2 (color online). Simulated dispersion relation for channeled and bulk spin waves. $\Omega_{k}^{u}$ indicate bulk modes. The channeled modes for Bloch-type $\left(\Omega_{k}^{B}\right)$ and Néel-type $\left(\Omega_{k}^{N \pm}\right)$ walls are computed, where $D=3 \mathrm{~mJ} / \mathrm{m}^{2}$ for the latter and the sign indicates propagation relative to the wall chirality. The points represent simulated values and lines are based on Eqs. (2), (3), and (4). The inset shows the three-domain geometry with two parallel domain wall channels.

magnetic state of the track comprises a three-domain structure, where the domain walls run approximately parallel to the track edges. In order to stabilize this particular domain state, a DMI of $D=1 \mathrm{~mJ} / \mathrm{m}^{2}$ was used to ensure that the domain walls are not expelled from the track as a result of dipolar interactions. A microwave antenna is placed at one end of the track, which excites spin waves that propagate in a counterclockwise direction along the track. Figure 3 illustrates the propagation for an excitation at $5 \mathrm{GHz}$, which is in the gap of the bulk modes. A clear channeling effect can be observed, where the spin waves can be seen to propagate along the curved track without any apparent scattering or loss of coherence (in contrast to bulk modes, as shown in Fig. S2 of Ref. [23]), and again there is no perceptible interference between the two domain wall channels. The nonreciprocal effect due to the different relative chiralities seen for the propagating modes is also preserved, which suggests that closely spaced domain walls act as independent channels for excitations in the frequency gap of the bulk modes. While difficult to realize in practice, this geometry serves to illustrate the salient features of domain wall channeling along curved paths. Note that the DMI is not necessary for propagation along curved walls; some examples for Bloch walls with $D=0$ can be found elsewhere [23].

The dispersion relation for the Néel wall modes has interesting consequences for wave packet propagation. In the ultrathin film geometry in which a strong perpendicular anisotropy is present, the bulk spin wave spectrum is mainly exchange dominated and exhibits a quadratic dispersion relation, as illustrated in Figs. 1(b) and 2. As such, wave packets comprising bulk spin waves in the long wavelength limit, $k \rightarrow 0$, exhibit strong dispersion and a vanishing 


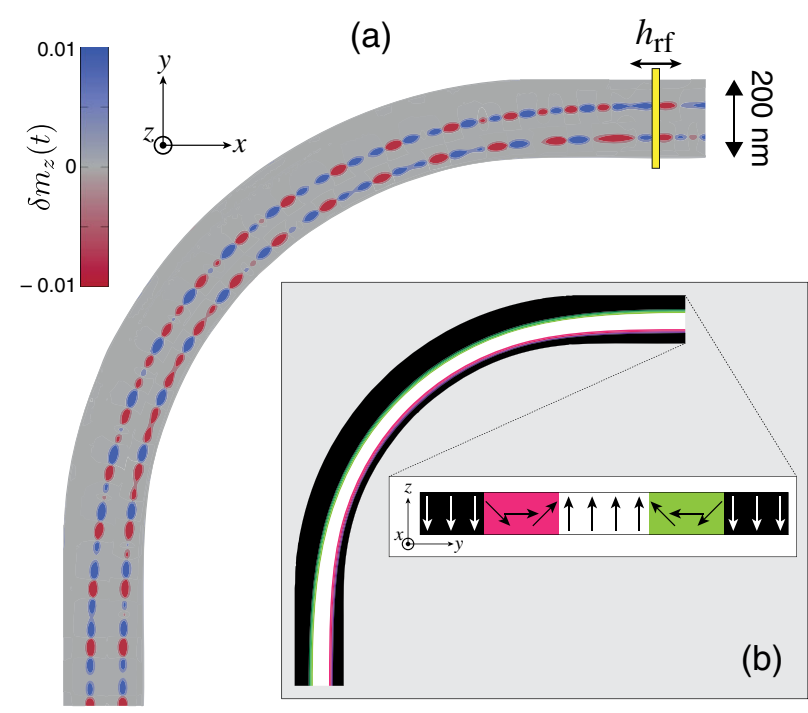

FIG. 3 (color online). (a) Spin wave channeling around a curved track through two Néel-type domain walls $\left(D=1 \mathrm{~mJ} / \mathrm{m}^{2}\right)$. Fluctuations in the $m_{z}$ magnetization component are shown as a color code for a microwave excitation frequency of $5 \mathrm{GHz}$, where the simulated microwave antenna is situated at the top of the track. The area of the simulated region is $1600 \mathrm{~nm} \times 1600 \mathrm{~nm}$. The width of the wire is $200 \mathrm{~nm}$ and the thickness is $1 \mathrm{~nm}$. (b) Equilibrium configuration of the curved track comprising three domains. The inset shows a schematic of the magnetization profile in a cross section at the top of the track.

group velocity, $v_{g}$, since $v_{g}^{u} \equiv \partial_{k} \Omega_{k}^{u}=4 \gamma A k / M_{s}$ is linear in $k$ and vanishes as $k \rightarrow 0$. For Bloch-type walls, $v_{g}$ for the channeled mode remains finite in this limit as a result of the weak ellipticity of the precession, with a value of $v_{q 0}^{B} \equiv v_{g}^{B}(k=0)=\gamma \sqrt{2 A K_{\perp}} / M_{s}$, which is approximately $76 \mathrm{~m} / \mathrm{s}$ with the numerical parameters considered here. For Néel-type walls, the channeled spin wave modes in the long wavelength limit possess a group velocity characterized by

$$
v_{g 0}^{N \pm}=\frac{\gamma}{M_{s}}\left(\sqrt{A\left(\frac{\pi D}{\lambda}-2 K_{\perp}\right)} \pm \frac{\pi D}{2}\right),
$$

which is found from Eq. (4). A striking feature is that the group velocity of the two branches can have large finite values even in the long wavelength limit, but which are strongly dependent on the propagation direction as shown in Fig. 4. The difference in $v_{g 0}^{N \pm}$ between the two branches is simply proportional to $D$, but the functional form of $v_{g 0}^{N \pm}(D)$ itself is nontrivial and is shown in the inset of Fig. 4(a). It is interesting to note that the group velocity for the $\Omega_{k}^{N-}$ branch tends towards zero as $D$ increases, which is mirrored by an increasing group velocity for the $\Omega_{k}^{N+}$ mode. For a moderate value $D=1.5 \mathrm{~mJ} / \mathrm{m}^{2}$, $v_{g 0}^{N+} \approx 1000 \mathrm{~m} / \mathrm{s}$, which might be a useful characteristic for information technologies.

An illustration of nonreciprocal wave packet propagation is shown in Fig. 4(b). The geometry shown in Fig. 1 is used. The wave packets are generated with a field pulse at $x=0$ that comprises a sine wave oscillation, $h_{p}=h_{p 0} \sin \left(2 \pi \nu_{p} t\right)$ with $h_{p 0}=100 \mathrm{mT}$ and $\nu_{p}=7.5 \mathrm{GHz}$, over one period. This form allows better wave vector selection in reciprocal space, since the Fourier transform of this function is peaked at a finite value of $k_{x}$. The wave packet is generated in a straight Néel-type wall [cf. Fig. 1(e)] with a large value of the DMI, $D=3 \mathrm{~mJ} / \mathrm{m}^{2}$, in order to highlight the nonreciprocity. The temporal evolution of the $\delta m_{z}$ component of the wave packet is shown for three instants after the application of the pulsed field. Propagation towards the $-x$ direction involves the $\Omega_{k}^{N-}$ branch and exhibits strong dispersion, where the wave packet spreads out over a micron after $1.5 \mathrm{~ns}$. On the other hand, the propagation along the $+x$ direction $\left(\Omega_{k}^{N+}\right)$ exhibits a much weaker dispersion where the wave packet can be observed to retain its shape after propagating over $2 \mu \mathrm{m}$. The decrease in amplitude for the $\Omega_{k}^{N+}$ packet is related to Gilbert damping ( $\alpha=0.01$ ), rather than dispersion. The wave packet velocity for $\Omega_{k}^{N+}$ computed from simulation is approximately $1550 \mathrm{~m} / \mathrm{s}$, which is close to the value of $\approx 1700 \mathrm{~m} / \mathrm{s}$ expected from the analytical theory. Good agreement for the velocity is also found for the $\Omega_{k}^{N-}$ branch,
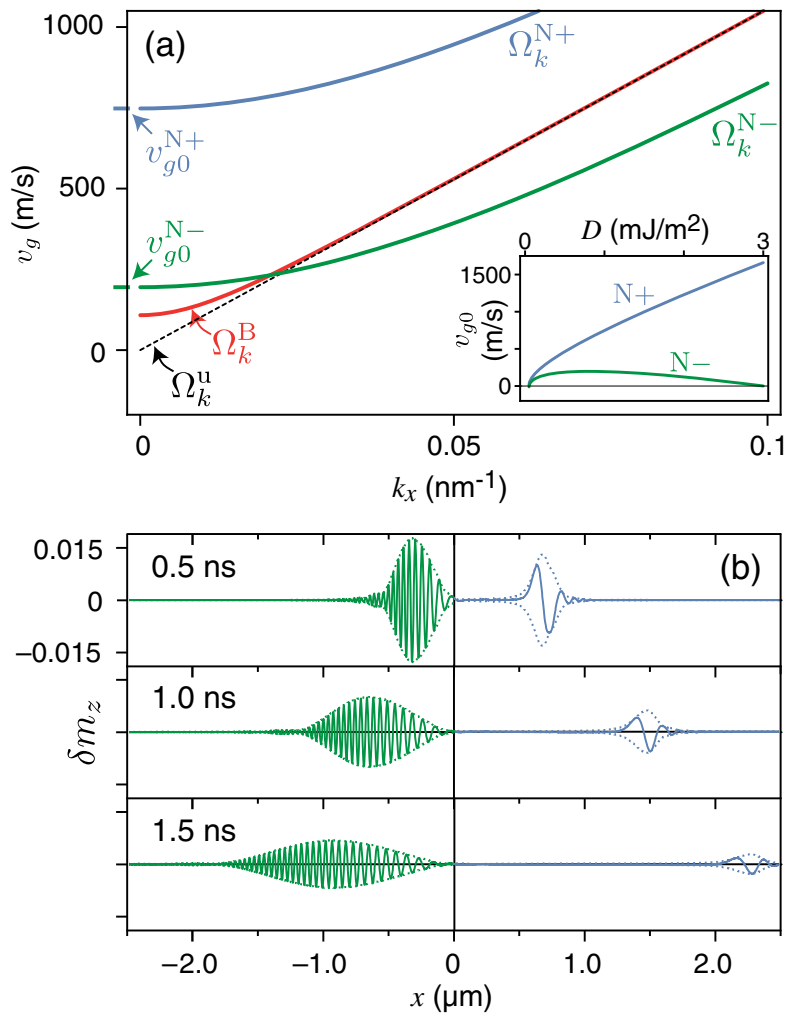

FIG. 4 (color online). (a) Group velocity for the channeled and bulk spin waves. $\Omega_{k}^{u}$ indicate bulk modes. The channeled modes for Bloch-type $\left(\Omega_{k}^{B}\right)$ and Néel-type $\left(\Omega_{k}^{N \pm}\right)$ walls $\left(D=1 \mathrm{~mJ} / \mathrm{m}^{2}\right)$ are computed, where the sign for the latter indicates propagation relative to the wall chirality. The inset shows the $k \rightarrow 0$ limit of the group velocity for the two Néel wall modes as a function of the Dzyaloshinskii-Moriya constant, $D$. (b) Wave packets in a Néel wall channel $\left(D=3 \mathrm{~mJ} / \mathrm{m}^{2}\right)$ at three instants after generation by a sinusoidal pulse with $\nu_{p}=7.5 \mathrm{GHz}$. 
where simulations give a value of $\approx 750 \mathrm{~m} / \mathrm{s}$ while the analytical theory predicts a group velocity of $\approx 775 \mathrm{~m} / \mathrm{s}$.

Domain wall channeling addresses some of the issues highlighted in the introduction for magnonics applications. Because the spin structure of domain walls is primarily governed by intrinsic magnetic properties, they are less sensitive to issues related to lithography or nanofabrication such as edge roughness or sample-to-sample reproducibility. Channeling along curved walls might also be useful for modulating propagation lengths for applications involving interference, but it would at the very least relax constraints on how straight spin wave conduits need to be for magnonic circuits based on such waveguides to function. Reconfigurable waveguide schemes could also be envisaged, where the number, spacing, and shape of domain wall arrays could be modified with applied fields or spin-polarized currents. On a more fundamental level, the crossing of the localized and bulk mode branches for finite $D$ suggests there are severe consequences on dissipation via spin wave interactions, and consequently their lifetimes, near this critical wave vector. We also suspect that the nonreciprocity has intriguing consequences on the flow of angular momentum, yet another aspect that may be distinctly different from whispering gallery modes in optical fields.

The authors acknowledge fruitful discussions with $\mathrm{M}$. Bailleul, Y. Henry, R. Hertel, T. Devolder, and S. PetitWatelot. This work was partially supported by the French National Research Agency (ANR) under Contract No. ANR11-BS10-0003 (NanoSWITI), the University of Glasgow, EPSRC (EP/M024423/1 [40]), and the National Council of Science and Technology of Mexico (CONACyT).

*felipe.garcia@u-psud.fr †joo-von.kim@u-psud.fr

[1] Lord Rayleigh, Philos. Mag. 20, 1001 (1910).

[2] T. Nobis, E. M. Kaidashev, A. Rahm, M. Lorenz, and M. Grundmann, Phys. Rev. Lett. 93, 103903 (2004).

[3] A. Khitun, M. Bao, and K. L. Wang, J. Phys. D 43, 264005 (2010).

[4] V. V. Kruglyak, S. O. Demokritov, and D. Grundler, J. Phys. D 43, 264001 (2010).

[5] A. A. Serga, A. V. Chumak, and B. Hillebrands, J. Phys. D 43, 264002 (2010).

[6] B. Lenk, H. Ulrichs, F. Garbs, and M. Münzenberg, Phys. Rep. 507, 107 (2011).

[7] Magnonics-From Fundamentals to Applications, edited by S. O. Demokritov and A. N. Slavin, Topics in Applied Physics Vol. 125 (Springer-Verlag, Berlin, 2013).

[8] R. Hertel, W. Wulfhekel, and J. Kirschner, Phys. Rev. Lett. 93, 257202 (2004).

[9] S.-K. Kim, J. Phys. D 43, 264004 (2010).

[10] K. Vogt, H. Schultheiss, S. Jain, J. E. Pearson, A. Hoffmann, S. D. Bader, and B. Hillebrands, Appl. Phys. Lett. 101, 042410 (2012).

[11] V.S. Tkachenko, A. N. Kuchko, M. Dvornik, and V. V. Kruglyak, Appl. Phys. Lett. 101, 152402 (2012).
[12] J. M. Winter, Phys. Rev. 124, 452 (1961).

[13] H.-B. Braun, Phys. Rev. B 50, 16485 (1994).

[14] C. Bayer, H. Schultheiss, B. Hillebrands, and R. L. Stamps, IEEE Trans. Magn. 41, 3094 (2005).

[15] A. K. Zvezdin and A.F. Popkov, JETP Lett. 39, 419 (1984).

[16] D. Bouzidi and H. Suhl, Phys. Rev. Lett. 65, 2587 (1990).

[17] Y. Le Maho, J.-V. Kim, and G. Tatara, Phys. Rev. B 79, 174404 (2009).

[18] A. V. Mikhaı̌lov and A. I. Yaremchuk, JETP Lett. 39, 354 (1984).

[19] D. Hinzke and U. Nowak, Phys. Rev. Lett. 107, 027205 (2011).

[20] P. Yan, X. S. Wang, and X. R. Wang, Phys. Rev. Lett. 107, 177207 (2011).

[21] X.-G. Wang, G.-H. Guo, Y.-Z. Nie, G.-F. Zhang, and Z.-X. Li, Phys. Rev. B 86, 054445 (2012).

[22] E. G. Tveten, A. Qaiumzadeh, and A. Brataas, Phys. Rev. Lett. 112, 147204 (2014).

[23] See Supplemental Material at http://link.aps.org/supplemental/ 10.1103/PhysRevLett.114.247206 for simulation details and additional simulation data, which includes Refs. [24, 25].

[24] http://mumax.github.io.

[25] T. Devolder, P. H. Ducrot, J.-P. Adam, I. Barisic, N. Vernier, J.-V. Kim, B. Ockert, and D. Ravelosona, Appl. Phys. Lett. 102, 022407 (2013).

[26] A. Vansteenkiste, J. Leliaert, M. Dvornik, M. Helsen, F. García-Sánchez, and B. Van Waeyenberge, AIP Adv. 4, 107133 (2014).

[27] A. Mougin, M. Cormier, J.-P. Adam, P. J. Metaxas, and J. Ferré, Europhys. Lett. 78, 57007 (2007).

[28] A. Fert and P. M. Levy, Phys. Rev. Lett. 44, 1538 (1980).

[29] A. Fert, Mater. Sci. Forum 59-60, 439 (1990).

[30] A. Crépieux and C. Lacroix, J. Magn. Magn. Mater. 182, 341 (1998).

[31] A. N. Bogdanov and U. K. Rößler, Phys. Rev. Lett. 87, 037203 (2001).

[32] M. Heide, G. Bihlmayer, and S. Blügel, Phys. Rev. B 78, 140403 (2008).

[33] A. Thiaville, S. Rohart, É. Jué, V. Cros, and A. Fert, Europhys. Lett. 100, 57002 (2012).

[34] J. P. Tetienne, T. Hingant, L. J. M. i. nez, S. Rohart, A. Thiaville, L. H. Diez, K. Garcia, J.-P. Adam, J.-V. Kim, J. F. Roch, I. M. Miron, G. Gaudin, L. Vila, B. Ocker, D. Ravelosona, and V. Jacques, Nat. Commun. 6, 6733 (2015).

[35] M. Belmeguenai, J.-P. Adam, Y. Roussigné, S. Eimer, T. Devolder, J.-V. Kim, S. M. Cherif, A. Stashkevich, and A. Thiaville, Phys. Rev. B 91, 180405(R) (2015).

[36] F. Garcia-Sanchez, P. Borys, A. Vansteenkiste, J.-V. Kim, and R. L. Stamps, Phys. Rev. B 89, 224408 (2014).

[37] L. Udvardi and L. Szunyogh, Phys. Rev. Lett. 102, 207204 (2009).

[38] K. Zakeri, Y. Zhang, J. Prokop, T.-H. Chuang, N. Sakr, W. X. Tang, and J. Kirschner, Phys. Rev. Lett. 104, 137203 (2010).

[39] J.-H. Moon, S.-M. Seo, K.-J. Lee, K.-W. Kim, J. Ryu, H.-W. Lee, R. D. McMichael, and M. D. Stiles, Phys. Rev. B 88, 184404 (2013).

[40] F. Garcia-Sanchez, P. Borys, R. Soucaille, J.-P. Adam, R. L. Stamps, and J.-V. Kim, 10.5525/gla.researchdata.170. 